\title{
Editorial
}

\section{Collectivity in High Energy Heavy-Ion Collisions}

\author{
Md. Nasim, ${ }^{1}$ Shusu Shi, ${ }^{2}$ Sandeep Chatterjee, ${ }^{3}$ Subhash Singha, ${ }^{4}$ and Victor Roy ${ }^{5}$ \\ ${ }^{1}$ University of California, Los Angeles, CA 90095, USA \\ ${ }^{2}$ Central China Normal University, Wuhan, China \\ ${ }^{3}$ AGH University of Science and Technology, 30-059 Krakow, Poland \\ ${ }^{4}$ Kent State University, Kent, OH 44240, USA \\ ${ }^{5}$ National Institute of Science Education and Research, Odisha 752050, India \\ Correspondence should be addressed to Md. Nasim; nasim@rcf.rhic.bnl.gov
}

Received 7 June 2017; Accepted 8 June 2017; Published 19 September 2017

Copyright (C) $2017 \mathrm{Md}$. Nasim et al. This is an open access article distributed under the Creative Commons Attribution License, which permits unrestricted use, distribution, and reproduction in any medium, provided the original work is properly cited. The publication of this article was funded by $\mathrm{SCOAP}^{3}$.

The primary purpose of the relativistic heavy-ion collisions experiment is to create QCD matter under high temperature and high density, Quark-Gluon Plasma (QGP), and study its properties. Experiments at RHIC and LHC have shown that a very dense QCD medium is formed in high energy heavy-ion collisions. The azimuthal anisotropy parameter $\left(v_{n}\right)$ has been considered as an excellent tool to study the system formed in the early stages of high energy heavy-ion collisions. Observed large magnitude of azimuthal anisotropy parameters (and observation of constituent quark scaling) in nucleus-nucleus collisions at RHIC has been considered as a signature of collectivity of the system formed after the collision. However, recent results from LHC show large deviation of the constituent quark scaling. In addition, the observation of nonzero $v_{2}$ in a small system ( $+\mathrm{p}, \mathrm{p}+\mathrm{A}, \mathrm{d}+\mathrm{A}$, and $\left.{ }^{3} \mathrm{He}+\mathrm{A}\right)$ at $\mathrm{LHC}$ and RHIC has triggered theoretical debate over our understating of collectivity. In this special issue, we collected articles (both overview and original papers) with the aim of a systematic study of collectivity in heavy-ion collisions and hence to understand properties and evolution of the QuarkGluon Plasma.

The paper "Collectivity in Small Collision Systems: An Initial-State Perspective" reviews the recent theoretical developments and outstanding issues about flow in collisions of small systems. This paper specifically focuses on theoretical explanations based on the initial-state dynamics. It has been shown that calculations based purely on initialstate correlations are able to describe various experimental observations in small collision system up to the highest multiplicity regions; however simultaneous description of the low and high $p_{T}$ observables over a wide range of multiplicity remains challenging.

The review article "Relativistic Hydrodynamics in HeavyIon Collisions: General Aspects and Recent Developments" presents the latest developments (event-by-event fluctuations, flow in small systems, flow in ultracentral collisions, longitudinal fluctuations, and correlations and flow in an intense magnetic field) in the hydrodynamical modeling of relativistic heavy-ion collisions. The effect of viscosity on particle spectra and anisotropic flow parameter has been discussed. It has been pointed out that the effect of magnetic field might not be negligible on the hydrodynamics evolution of QGP produced in heavy-ion collisions. This paper also discussed that the origin of collectivity in the small system is still not well understood.

A detailed review on heavy flavor elliptic flow $\left(v_{2}\right)$ from the experimental point of view has been discussed in "An Experimental Review on Heavy-Flavor in HeavyIon Collision." This article reviews several important results from RHIC and LHC experiments and discussed their implications. This review article focused on measurements of heavy-flavor as a function of $p_{T}$, collision centrality, and energy carried out in RHIC and LHC experiments. The comparison of these experimental measurements with available theoretical model predictions is also shown. Simultaneous description of the measured $v_{2}$ and nuclear modification factor $\left(R_{\mathrm{AA}}\right)$ by a theoretical model is a challenging job and is an open issue. It has been pointed out that the current heavyflavor measurements are limited by statistics. The Heavy Flavor Tracker (HFT) at STAR and upgraded Inner Tracker 
System (ITS) at ALICE are expected to allow for a precise measurement in future.

The paper "Spectra and Elliptic Flow of (Multi)Strange Hadrons at RHIC and LHC within Viscous Hydrodynamics + Hadron Cascade Hybrid Model" discusses the spectra and elliptic flow of (multi)strange hadrons at RHIC and LHC using VISHNU model. The VISHNU is a hybrid model with $(2+1)$-dimensional ultrarelativistic viscous hydrodynamics + hadron cascade. It has been shown that the VISHNU model gives good descriptions of the spectra and elliptic flow of these strange and multistrange hadrons at several centrality classes at RHIC and LHC.

The review article "Review of Anisotropic Flow Correlations in Ultrarelativistic Heavy-Ion Collisions" presents recent developments of correlations of anisotropic flow harmonics. Event-by-event measurement of anisotropic flow is shown to be crucial to understand the initial conditions in heavy-ion collisions. Correlations between different order flow harmonics and magnitude of flow fluctuation are predicted to be sensitive to the transport properties of the produced medium in heavy-ion collisions.

The paper "A Universal Description of Pseudorapidity Distributions in Both Nucleus-Nucleus and p-p Collisions at Currently Available Energies" presents a description of relativistic hydrodynamics model which describes the pseudorapidity distributions of charged particles measured in both nucleus-nucleus and $\mathrm{p}$-p collisions at currently available energies. In this model, the collective motion is assumed to obey the hydrodynamic model which integrates the features of Landau and Hwa-Bjorken theory.

The paper "Beam Energy Dependence of HanburyBrown-Twiss Radii from a Blast-Wave Model" discusses the beam energy dependence of correlation lengths (the Hanbury-Brown-Twiss radii) which is calculated by using a Blast-Wave model. The transverse momentum dependence of $\mathrm{HBT}$ radii is presented for $\mathrm{Au}+\mathrm{Au}$ collision at $200 \mathrm{GeV}$ and for $\mathrm{Pb}+\mathrm{Pb}$ collisions at $2.76 \mathrm{TeV}$. This paper shows that particle emission duration cannot be ignored while calculating the HBT radii with the same parameters.

The review article "A Review of Elliptic Flow of Light Nuclei in Heavy-Ion Collisions at RHIC and LHC Energies" presents a review of the measurements of the elliptic flow of light nuclei (d, d-bar, t ${ }^{3} \mathrm{He}$, and ${ }^{3} \mathrm{He}$-bar) from the RHIC and LHC experiments. Comparison between light (anti)nuclei $v_{2}$ has been compared with that of the (anti)proton. A mass ordering in $v_{2}$ between nuclei and other hadrons is observed. A prediction of $v_{2}$ for ${ }^{3} \mathrm{He}$ and ${ }^{4} \mathrm{He}$ using coalescence and blast-wave models is also shown.

Overall, we are very hopeful that the theoretical and experimental issues covered here will impact the future of heavy-ion physics and lead to further studies that will help us to understand more about the origin of collectivity.

$$
\begin{array}{r}
\text { Md. Nasim } \\
\text { Shusu Shi } \\
\text { Sandeep Chatterjee } \\
\text { Subhash Singha } \\
\text { Victor Roy }
\end{array}
$$



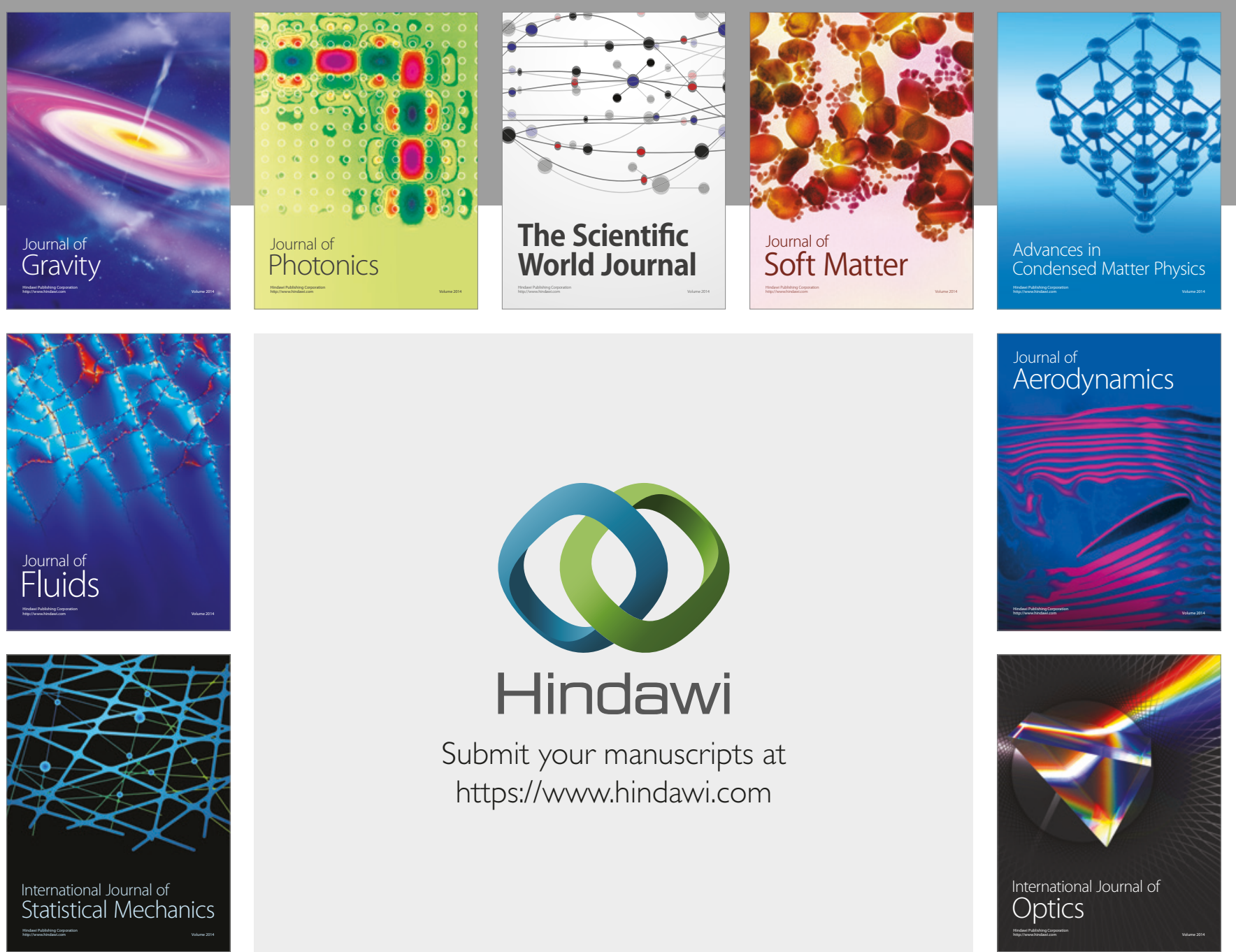

Submit your manuscripts at

https://www.hindawi.com
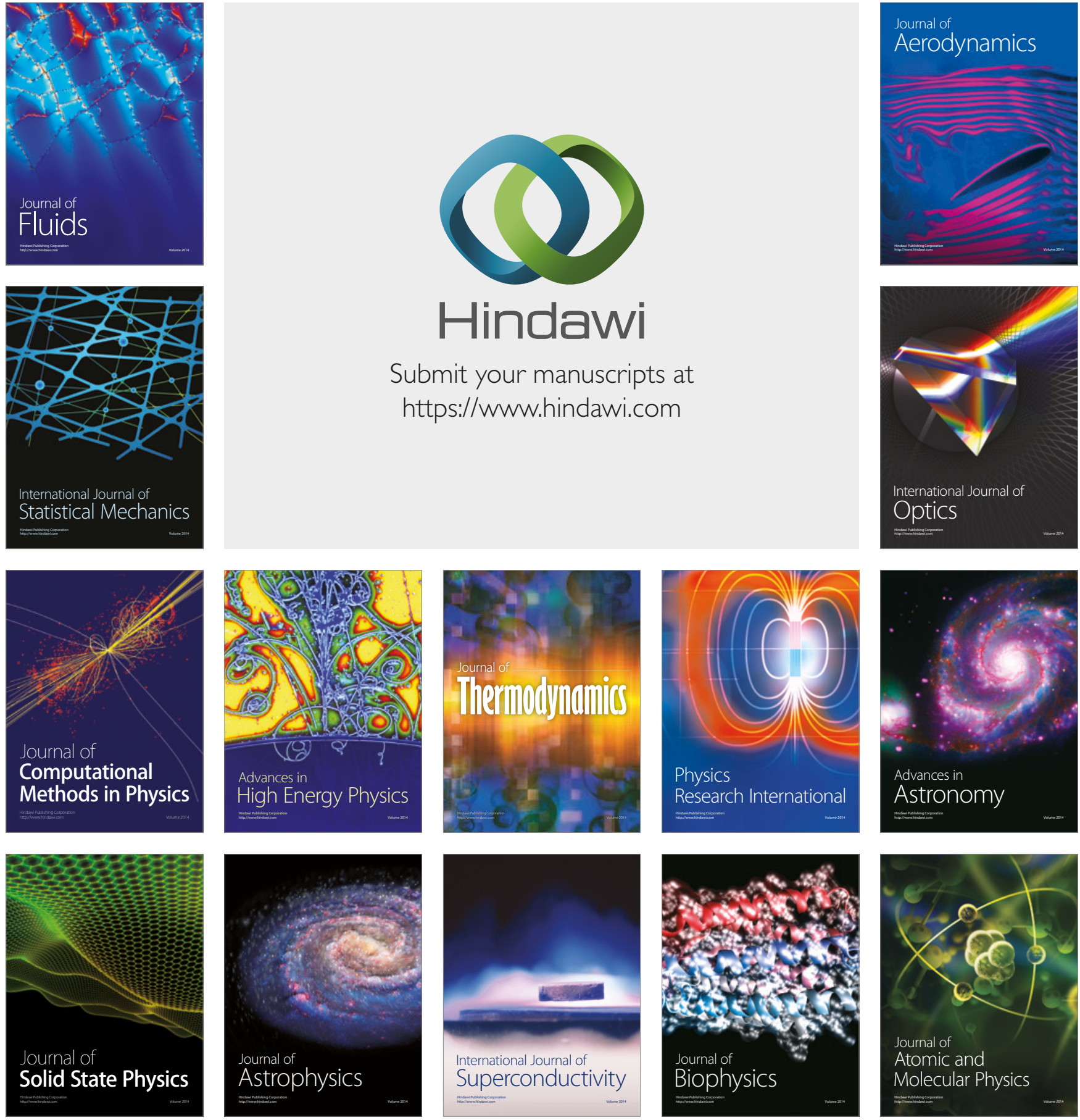